\title{
Rayleigh Fading Multiple Access Channel without Channel State Information
}

\author{
Ninoslav Marina \\ Mobile Communications Laboratory (LCM) \\ School of Computer and Communication Sciences \\ Swiss Federal Institute of Technology (EPFL) \\ CH-1015 Lausanne, Switzerland \\ firstname.lastname@epfl.ch
}

\begin{abstract}
In this paper we determine bounds of the capacity region of a two-user multiple-access channel with Rayleigh fading when neither the transmitters nor the receiver has channel state information (CSI). We assume that the fading coefficients as well as the additive noise are zero-mean complex Gaussian and there is an average power constraint at the channel input for both senders. Results that we get show that the lower (inner) and the upper (outer) bound of the capacity region are quite close for low and high signal-to-noise ratio (SNR). Surprisingly, the boundary of the capacity region is achieved by time sharing among users, which is not the case for fading channels with perfect CSI at the receiver. As an additional result we derive a closed form expression for the mutual information if the input is on-off binary.

Index Terms - Multiple-access channel, capacity region, Rayleigh fading, channel state information, volume of the capacity region.
\end{abstract}

\section{Introduction}

Wireless communication systems are currently becoming more and more important. A challenging task for operators of mobile communication systems and researchers is the need to constantly improve spectral efficiency, maintain a desirable quality of service, minimize the consumption of transmit power in order to lower electromagnetic radiation and prolong the battery life. In the same time the number of base stations has to be minimized, whilst accommodating as many users as possible. In fulfilling these requirements, the greatest obstacle is the nature of the mobile communication channel, which is time-varying, due to rapid changes in the environment and mobility of users. Signal strength may drop by several orders of magnitude due to an increase in distance between transmitter and receiver and superposition phenomena in scattering environments. This phenomenon is commonly known as fading and such channels as fading channels. Many modern wireless systems send a training sequence inserted in the data stream in order to provide the receiver with information about the channel. On the other hand, some systems provide a feedback channel from the receiver to the transmitter and this information can help the transmitter to choose an appropriate signal to access the channel. Knowledge of the channel is known as 
channel state information (CSI). Many papers have been written on channels with perfect CSI at the receiver, at the transmitter, at both and at neither of them. In practical wireless communication systems, whenever there is a large number of independent scatterers and no line-of-sight path between the transmitter and the receiver, the radio link may be modelled as a Rayleigh fading channel. In a multi-user environment the uplink channel is typically modelled as multiple access channel (MAC). The performance of the channel strongly depends on the fact whether the state of the channel is available at the receiver and(or) the transmitter(s). In this paper we are interested in deriving the capacity region of the two-user Rayleigh fading channel without CSI at the receiver or the transmitter. The capacity region of a multiple access channel is the closure of achievable rates for all users [5]. This channel is of interest since in some cases the channel can vary very quickly and it will be not possible to send any information about the channel.

The case without channel state information for the single user channel has been studied in $[9,7,3,6]$ and for the multi-user channel in [8,2]. In [3], authors show that without channel state information, the optimal input is discrete with a mass point at zero. In [9] it is shown that without channel state information, the capacity at high SNR depends double-logarithmically on the SNR. A more general result on the double logarithmic behavior at high SNR is given in [6].

In Section 2 we establish a closed form solution for the mutual information when the input is binary on-off. In Section 3 we find lower and upper bounds of the capacity region of a two-user Rayleigh fading channel. We compare the bounds in Section 4 and give conclusions in Section 5.

\section{Rayleigh Fading Channels without CSI}

The capacity $C_{s u}(\rho)$ of the single user channel $Y^{\prime}=A X^{\prime}+Z$, where $A, Z \sim$ $\mathcal{N}_{\mathbb{C}}(0,1)$ and $E\left[|X|^{2}\right] \leq \rho$ has been derived in [3]. Having $\rho=\sigma_{A}^{2} P / \sigma_{Z}^{2}$, the capacity of this channel is the same as the capacity of the channel with $A \sim$ $\mathcal{N}_{\mathbb{C}}\left(0, \sigma_{A}^{2}\right), Z \sim \mathcal{N}_{\mathbb{C}}\left(0, \sigma_{Z}^{2}\right)$ and $E\left[|X|^{2}\right] \leq P$. According to [3], the capacity achieving input distribution is discrete. For low SNR, the mutual information for binary inputs is not far from the capacity. For extremely high SNR, higher than the fading number, defined in [6], the capacity behaves as $\log (\log (\mathrm{SNR}))$.

Next we give a closed form expression for the mutual information between the input and the output, if the input is on-off binary, namely $(0, b)$. The binary on-off input is interesting since for low SNR, it is optimal. At the end of the section we give a numerical result for the capacity.

Proposition 1. (Closed form expression for the mutual information for a particular on-off input probability $p$ and SNR $\rho$ ): For the channel $Y=A X+Z$, when the input is binary on-off with $\operatorname{Pr}\{X=0\}=1-p$ and power constraint $E\left[|X|^{2}\right] \leq \rho$, the mutual information between the input $X$ and the output $Y$, is

$$
I(X ; Y)=\mathrm{h}(p)+p \mathcal{J}\left(\frac{p+\rho}{p^{2}(1-p)^{-1}}, \frac{\rho}{p}\right)+(1-p) \mathcal{J}\left(\frac{p^{2}(1-p)^{-1}}{p+\rho}, \frac{\rho}{p+\rho}\right)(1)
$$


where $\mathrm{h}(\cdot)$ is the binary entropy function, $\mathcal{J}(c, d)=-\ln (1+c)+\frac{c d}{1+d} \cdot{ }_{2} F_{1}(1,1+$ $\left.d^{-1} ; 2+d^{-1} ;-c\right)$, and ${ }_{2} F_{1}(u, v ; w ; z)=\frac{\Gamma(w)}{\Gamma(u) \Gamma(v)} \sum_{k=0}^{\infty} \frac{\Gamma(u+k) \Gamma(v+k)}{\Gamma(w+k)} \cdot \frac{z^{k}}{k !}$ is the Gaussian hypergeometric function defined in [4]. $\Gamma(q)=\int_{0}^{\infty} x^{q-1} e^{-x} d x$ is the Euler gamma function.

The detailed proof is given in [1].

To compute the capacity of the binary input Rayleigh fading channel without channel state information, denoted by $C_{b}$, one has to find the maximum of $I_{p, \rho}(X ; Y)$ over $p$ for different $\rho$. Unfortunately $d I_{p, \rho}(X ; Y) / d p=0$ is a transcendental equation and cannot be solved explicitly. The capacity and the optimizing $p^{*}$ as functions of $\rho$ are shown in Fig. 1. Note that as the power of the input signal increases the information rate of this channel goes to its limit of $\ln 2$ nats and $p^{*}$ goes to 0.5. This happens since if $\rho$ goes to $\infty$, we get the channel $Y=A X$.
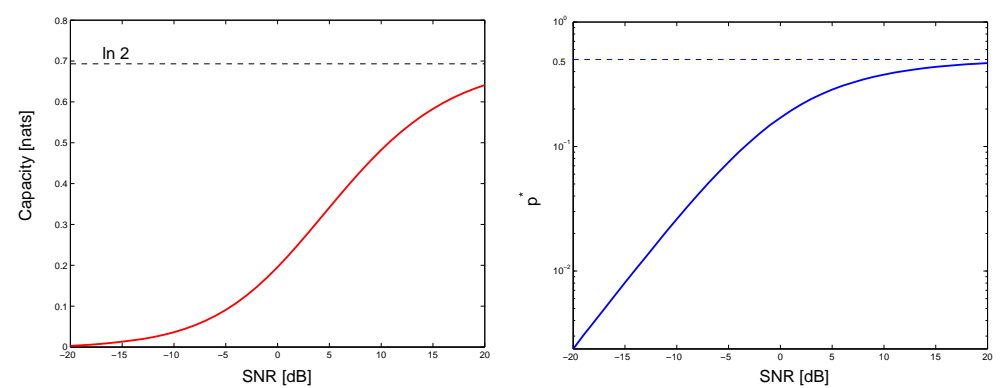

Figure 1. Capacity $C_{b}$ and the optimal probability $p^{*}$ as function of $\rho$

\section{Two-User Rayleigh Fading Channel}

In this section, we give a lower and an upper bound of the capacity region of a two-user Rayleigh fading channel in the case where the channel is not known either at the transmitters or at the receiver, but all of them know the statistics of the channel exactly. The ratio of volumes [1] of the lower and the upper bound of the capacity region will serve us as a measure for the proximity of these bounds. The channel is

$$
\tilde{Y}=\tilde{A}_{1} \tilde{X}_{1}+\tilde{A}_{2} \tilde{X}_{2}+\tilde{Z},
$$

where $\tilde{A}_{1}, \tilde{A}_{2}$ and $\tilde{Z}$ are independent and identically distributed (i.i.d.), zeromean, complex Gaussian random variables of variances $\sigma_{A_{1}}^{2}, \sigma_{A_{2}}^{2}$ and $\sigma_{Z}^{2}$ respectively. There are input constraints, $E\left[\left|\tilde{X}_{1}\right|\right]^{2} \leq P_{1}$ and $E\left[\left|\tilde{X}_{2}\right|\right]^{2} \leq P_{2}$. No channel information is provided to the transmitters and the receiver. However, they perfectly know the statistics of the channel. To find "good" bounds of the capacity 
region of this channel, we use the results for the single user memoryless Rayleigh fading channel, studied in [3]. Channel (2) has the same capacity as the channel $\frac{\tilde{Y}}{\sigma_{Z}}=\frac{\tilde{A}_{1}}{\sigma_{A_{1}}} \frac{\sigma_{A_{1}}}{\sigma_{Z}} \tilde{X}_{1}+\frac{\tilde{A}_{2}}{\sigma_{A_{2}}} \frac{\sigma_{A_{2}}}{\sigma_{Z}} \tilde{X}_{2}+\frac{\tilde{Z}}{\sigma_{Z}}$. Letting $Y=\tilde{Y} / \sigma_{Z}, X_{1}=\sigma_{A_{1}} \tilde{X}_{1} / \sigma_{Z}$ and $X_{2}=\sigma_{A_{2}} \tilde{X}_{2} / \sigma_{Z}$, we get

$$
Y=A_{1} X_{1}+A_{2} X_{2}+Z
$$

with $A_{1}=\tilde{A}_{1} / \sigma_{A_{1}}, A_{2}=\tilde{A}_{2} / \sigma_{A_{2}}$ and $Z=\tilde{Z} / \sigma_{Z}$ all being $\mathcal{N}_{\mathbb{C}}(0,1)$. Power constraints on the new inputs become $E\left[\left|X_{1}\right|\right]^{2} \leq \sigma_{A_{1}}^{2} P_{1} / \sigma_{Z}^{2}=\rho_{1}$ and $E\left[\left|X_{2}\right|\right]^{2} \leq$ $\sigma_{A_{2}}^{2} P_{2} / \sigma_{Z}^{2}=\rho_{2}$. It is clear that doing these transforms, all mutual information in the new channel remain the same. Thus, the capacity region of the channel (2) is the same as the capacity region of the channel (3). For a particular input distribution, the region of achievable rates for the channel (3) is $\mathcal{R}\left(p_{X_{1}}, p_{X_{2}}\right)=\left\{\left(R_{1}, R_{2}\right) \in \mathbb{R}_{+}^{2}: R_{1} \leq I\left(Y ; X_{1} \mid X_{2}\right) ; R_{2} \leq I\left(Y ; X_{2} \mid X_{1}\right) ; R_{1}+\right.$ $\left.R_{2} \leq I\left(Y ; X_{1}, X_{2}\right)\right\}$. The capacity region is a closure of the convex hull of the union over all possible product input distributions $p_{X_{1}}(x) p_{X_{2}}(x)$ of all such regions $\mathcal{R}\left(p_{X_{1}}, p_{X_{2}}\right)$. To compute the maximum mutual information in the capacity region for user 1 and user 2 separately, we need to analyze the single user fading channel, similarly as it is done in [3]. Given $X_{2}=x_{2}$, the equivalent channel is $Y=A_{1} X_{1}+\left(A_{2} x_{2}+Z\right)$. This channel is the same as the single user fading channel $Y=A_{1} X_{1}+Z$, with larger variance of the additive noise, that is, $1+\left|x_{2}\right|^{2}$. Thus, it behaves as the channel $Y=A_{1} X_{1}+Z$, with different SNR constraint, that is, $\rho^{\prime}=\rho /\left(1+|x|^{2}\right)$. It is shown in [3] that the capacity achieving input distribution for this channel has to be discrete with a mass point at the origin. Moreover, it is shown in the same paper that for low SNR, the maximizing input distribution is binary. Thus, the rate of user 1 is bounded by $R_{1} \leq \sum_{x_{2}} p_{X_{2}}\left(x_{2}\right) I\left(X_{1} ; Y \mid X_{2}=\right.$ $\left.x_{2}\right) \leq \sum_{x \in \mathcal{X}_{2}} p_{X_{2}}(x) C_{s u}\left(\frac{\rho_{1}}{1+|x|^{2}}\right) \leq \sum_{x \in \mathcal{X}_{2}} p_{X_{2}}(x) C_{s u}\left(\rho_{1}\right)=C_{s u}\left(\rho_{1}\right)$, where the last inequality is achieved with equality if $p_{X_{2}}(0)=1$, i.e. if user 2 is silent. By $C_{s u}(\rho)$ we denote the capacity of the single user fading channel with no channel state information, for a particular $\mathrm{SNR}=\rho$. Thence, the point $C_{s u}\left(\rho_{1}\right)$ is achievable and it is the highest rate that can be achieved by user 1 , using the channel while user 2 is silent. That is one point on the boundary of the capacity region, namely the extreme point on the $R_{1}$-axis. From symmetry, the same is true for user 2, i.e. the extreme point on the $R_{2}$-axis is $C_{s u}\left(\rho_{2}\right)$.

After finding both extreme points, let us find the maximum sum rate. It is shown in [8] that if the propagation coefficients take on new independent values for every symbol (i.i.d.), then the total throughput capacity for any number of users larger than 1 , is equal to the capacity if there is only one user. Hence, time division multiple access (TDMA) is an optimal scheme for multiple users. In that case the sum rate is given by $\Theta=a C_{s u}\left(\rho_{1} / a\right)+(1-a) C_{s u}\left(\rho_{2} /(1-a)\right) \leq$ $C_{s u}\left(\rho_{1}+\rho_{2}\right)$, with $a \in[0,1]$. Note that the maximum throughput cannot be larger than $C_{s u}\left(\rho_{1}+\rho_{2}\right)$, the capacity which is achieved if both users fully cooperate, and is equivalent to the single user capacity for $\mathrm{SNR}=\rho_{1}+\rho_{2}$. The latest is achieved with equality for $a=\rho_{1} /\left(\rho_{1}+\rho_{2}\right)$. This is an upper bound of the capacity region, namely the pentagon $\left\{\left(R_{1}, R_{2}\right) \in \mathbb{R}_{+}^{2}: R_{1} \leq C_{s u}\left(\rho_{1}\right), R_{2} \leq\right.$ 
$C_{s u}\left(\rho_{2}\right), R_{1}+R_{2} \leq C_{s u}\left(\rho_{1}+\rho_{2}\right)$ (Fig. 2). A straightforward lower bound is

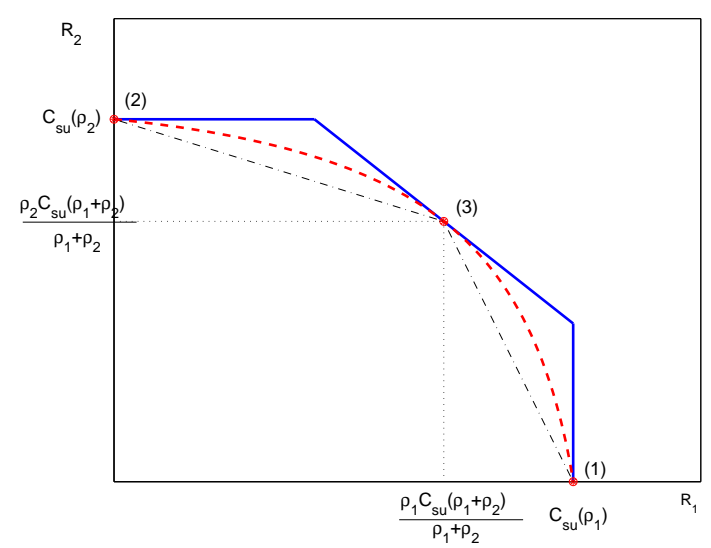

Figure 2. Lower and upper bounds of the capacity region, for given $\rho_{1}$ and $\rho_{2}$.

the region obtained by connecting the points that are achievable (dash-dot line in Fig. 2). Better lower bound is the time-sharing region (dashed line in Fig. 2 ). It is obtained by allowing user 1 to use the channel $a T$ seconds and user 2, $(1-a) T$ seconds. Because of the average power constraint the power used during the active period is normalized. Hence, the proposed lower bound of the capacity region parameterized by $a \in[0,1]$ is given by

$$
\begin{aligned}
& R_{1}(a)=a \cdot C_{s u}\left(\rho_{1} / a\right) \\
& R_{2}(a)=(1-a) \cdot C_{s u}\left(\rho_{2} /(1-a)\right) .
\end{aligned}
$$

The capacity region touches the upper bound in the following three points $\left(C_{s u}\left(\rho_{1}\right), 0\right),\left(0, C_{s u}\left(\rho_{2}\right)\right)$ and $\left(\frac{\rho_{1} C_{s u}\left(\rho_{1}+\rho_{2}\right)}{\rho_{1}+\rho_{2}}, \frac{\rho_{2} C_{s u}\left(\rho_{1}+\rho_{2}\right)}{\rho_{1}+\rho_{2}}\right)$. Note that a trivial upper bound that is much looser is the capacity region of the same channel, with perfect channel state information at the receiver.

\section{Comparison}

Comparing the bounds for different SNRs [1], it can be seen that for low and high SNR they are closer then for the some medium SNR. How to measure the "tightness" of the bounds? We propose comparing the volumes of the corresponding regions. For the two user case the volume is $\mathcal{V}_{2}=\int_{R_{1}} R_{2} d R_{1}$. It is easy to compute the volume of the upper bound in terms of the single-user capacities. For $\rho_{1}=\rho_{2}=\rho, \mathcal{V}_{U B}(\rho)=\frac{1}{2} \cdot\left[C_{s u}(2 \rho)\right]^{2}-\left[C_{s u}(2 \rho)-C_{s u}(\rho)\right]^{2}$. The volume of the lower bound is $\mathcal{V}_{L B}(\rho)=\int_{0}^{C_{s u}(\rho)} R_{2} d R_{1}=\int_{0}^{1} R_{2}(a) \dot{R}_{1}(a) d a$, where $R_{1}(a)$ and $R_{2}(a)$ are given by (4), and $\dot{R}_{1}(a)$ is the first derivative of $R_{1}$ with respect to 
$a$. It can be seen that for low SNR the lower and the upper bound are very close and as the SNR increases they diverge up to some SNR $(\sim 3 \mathrm{~dB})$, where the lower and the upper bound are at maximum "distance". In this case the ratio $\mathcal{V}_{L B} \simeq 0.925 \mathcal{V}_{U B}$. As SNR increases above $3 \mathrm{~dB}$, the bounds approach again, i.e. the ratio of $\mathcal{V}_{L B}$ and $\mathcal{V}_{U B}$ increases and tends to 1 . The results can be easily extended for an $M$-user case.

\section{Conclusions}

The single user Rayleigh fading channel with no side information has attracted some attention since it is useful for modelling different wireless channels. In this paper we get some insight for the multiple access Rayleigh fading channel with no CSI. We give bounds of the capacity region of the two-user Rayleigh multiple access channel. We see that the sum rate is maximized by time-sharing among users and in that case we achieve the boundary of the capacity region by giving to each user an amount of time that is proportional to its input average power constraint multiplied by the variance of the fading. This is not the case with multiple access channels with perfect CSI at the receiver only. However it is the case with the Gaussian MAC. We also see that the inner bound is always within $92.6 \%$ (in terms of the volume of the capacity region) of the outer bound. As an open problem for future research we leave the improvement of the inner and the outer bound.

\section{References}

1. N. Marina, "Successive Decoding," Ph.D. thesis, School of Computer and Communication Sciences, École Polytechnique Fédérale de Lausanne (EPFL), Lausanne 2004 .

2. N. Marina, "On successive decoding without channel state information," in Proc. IEEE Int. Symp. Telecommunications, Tehran, 2001.

3. I.C. Abou-Faycal, M.D. Trott and S. Shamai (Shitz), "The capacity of discrete-time memoryless Rayleigh-fading channels," IEEE Trans. on Information Theory, Vol. 47, No.4, May 2001.

4. M. Abramowitz and I.A. Stegun, Handbook of Mathematical Functions. New York: Dover, 1965.

5. T. M. Cover and J.A. Thomas, Elements of Information Theory. New York: Wiley, 1991.

6. A. Lapidoth and S.M. Moser, "Capacity bounds via duality with applications to multi-antenna systems on flat fading channels," IEEE Trans. on Information Theory, Vol. 49, No.10, Oct. 2003..

7. T. Marzetta and B. Hochwald, "Capacity of a mobile multiple-antenna communication link in Rayleigh flat fading," IEEE Trans. on Information Theory, Vol. 45, No.1, Jan. 1999.

8. S. Shamai (Shitz) and T.L. Marzetta, "Multiuser capacity in block fading with no channel state information," IEEE Trans. on Information Theory, Vol. 48, No.4, Apr. 2002.

9. G. Taricco and M. Elia, "Capacity of fading channel with no side information," Electronic Letters, vol. 33, no. 16, Jul. 1997. 